TITLE:

\title{
Analysis of the mandibular movement by simultaneous multisection continuous ultrafast MRI
}

\section{AUTHOR(S):}

Azuma, Takashi; Ito, Jin; Kutsuki, Manabu; Nakai, Ryusuke; Fujita, Shigeyuki; Tsutsumi, Sadami

\section{CITATION:}

Azuma, Takashi ...[et al]. Analysis of the mandibular movement by simultaneous

multisection continuous ultrafast MRI. Magnetic Resonance Imaging 2009, 27(3): 423-433

\section{ISSUE DATE:}

2009-04

URL:

http://hdl.handle.net/2433/87296

\section{RIGHT:}

c 2009 Elsevier Inc. All rights reserved.; この論文は出版社版でありませ ん。引用の際には出版社版をご確認ご利用ください。; This is not the published version. Please cite only the published version. 
Analysis of the mandibular movement by simultaneous multi-section continuous ultrafast MRI

Takashi Azuma ${ }^{1}$,Jin Ito $^{2}$,Manabu Kutsuki, ${ }^{3}$, Ryusuke Nakai ${ }^{1}$,Shigeyuki Fujita ${ }^{4}$, Sadami Tsutsumi ${ }^{1}$

${ }^{1}$ Research Center for Nano Medical Engineering, Institute for Frontier Medical Sciences, Kyoto University

${ }^{2}$ Department of Radiology, Kyoto Breast Center Sawai Clinic, Kyoto, Japan

${ }^{3}$ Ikenaga Dental Office, Osaka, Japan

${ }^{4}$ Department of Dentistry and Oral and Maxillofacial Surgery, Wakayama Medical University, Wakayama, Japan

Contact:azuma@frontier.kyoto-u.ac.jp 


\section{Summary}

The temporomandibular joint is the only bilaterally coupled joint in the human body. Thus, in dynamic MRI examination, both the right and left joints should be imaged simultaneously. We simultaneously imaged and analyzed the movement of both temporomandibular joints and mandibular movement using optimized ultrafast sequential imaging with multiple coils. 


\section{Abstract \\ Purpose:}

Using simultaneous multi-cross sectional imaging, four cross sections, including the mandibular midline and the right and left temporomandibular joints, were imaged to observe one movement of mouth opening and closing and analyze the movement of the mandible and temporomandibular joints.

Materials and Methods:

Four cross sections, including the midsagittal section of the mandible, the sagittal sections of the right and left temporomandibular joints and the horizontal section containing the heads of right and left temporomandibular joints, were imaged simultaneously. The imaging was conducted in ten male and female volunteers.

Results:

In all volunteers, the relationship of the mandibular movement on the median line with the right and left temporomandibular joints was observed. Images of the volunteers with trismus indicated the condition in which the right and left temporomandibular joints did not move in keeping with each other but moved independently from each other.

Conclusion:

Complex movement of the temporomandibular joint was first evaluated by simultaneous multiple-cross sectional MRI for the movement of mandible and temporomandibular joints. 


\section{Introduction}

The mandibular movement was conventionally measured by positioning indices on the skull surface; i.e., the movement of the temporomandibular joints was measured indirectly. This method can directly measure a tracing of the incisor point but not the mandibular movement [1] [2] [3] [4].

In contrast, MRI provides observation of any section and images of the articular disk soft tissue without radiation exposure and is very effective for observing the temporomandibular joints [5] [6]. MRI provides semi-movement images of articular tissues, but not continuous images. On opening and closing of the mouth (mandible) halfway, MR images are taken in several steps under the condition that the volunteers stop for several minutes with a spacer in their mouth. Based on a series of these static images, the movement can be measured [7] [8]. However, this cannot be considered to be an actual reproduction of movement. We therefore continuously visualized one side of the temporomandibular joints in opening and closing of the mouth by ultrafast imaging (Turbo-FLASH) in analyzable resolution and in real time and we used this data to measure the movement of the temporomandibular joint [9]. In many recent studies the movement of the temporomandibular joint has been imaged directly using MRI [10] [11] [12, 13], however, all of them examined only one side of the temporomandibular joint.

However, the temporomandibular joint is the only bilaterally coupled joint in the human 
body. Thus, temporomandibular joint dysfunction on one side can have an effect on the joint on the other side, resulting in an influence on the movement of the mandibular head $[4,14$, 15] [16].

Therefore, it is difficult to determine the relationship between the right and left movement when the movement of the respective temporomandibular joints is imaged separately. To accurately evaluate mandibular function, it is necessary to simultaneously image the movement of both the right and left temporomandibular joints; i.e., not independently. In particular, when the temporomandibular joint is impaired, the right and left joints move separately. Therefore, it is necessary to understand the relationship between simultaneous movement of the right and left temporomandibular joints. In this study, ultrafast sequential imaging was optimized and in addition to the sagittal section images of the right and left temporomandibular joints, the horizontal section images containing the heads of the right and left temporomandibular joints were also obtained; i.e., two kinds of sections of the right and left temporomandibular joints were imaged. Furthermore, the movement of the midsagittal section of the mandible was combined, i.e., four kinds of sections were imaged simultaneously. The relationship of measurements of the mandible and temporomandibular joints (hard tissues) with the articular disk and masticatory muscle (soft tissue) was examined. 


\section{Materials and Methods}

1. Dynamic MRI examination of temporomandibular joints

[Volunteers]

Ten male and female volunteers, aged from 22 to 40 years, underwent the examination.

After the study had been fully explained, written informed consent was obtained from all volunteers. Each volunteer was positioned supine on the MRI table and the maximal opening and closing movement of the mandible was imaged.

[MR system]

MR imaging was conducted using MAGNETOM Symphony Sonata 1.5T (SIEMENS, performance gradients: $40 \mathrm{mT} / \mathrm{m}$ maximum amplitude, $200 \mathrm{mT} / \mathrm{m} / \mathrm{msec}$ slew rate) with head, double-loop and neck coils. The movement of the right and left temporomandibular joints was imaged using the double-loop coil and the mandibular movement on the sagittal section was imaged using the neck coil.

[Imaging conditions]

True FISP (fast imaging with steady state precession) of ultrafast imaging sequence was optimized. To observe detailed movement of the mandible, the imaging time was shortened as 
much as possible. The sequence imaging time depends on the repetition time (TR), i.e., to increase a band width (BW) makes the TR shorter. Therefore, a decreased TR reduces the phase error associated with less binding artifacts. In this study, imaging was had a trial of as BW was decreased gradually from $1502 \mathrm{~Hz} / \mathrm{Pix}$, and finally the optimal BW for imaging of the temporomandibular joints was established to be $305 \mathrm{~Hz} / \mathrm{Pix}$, which provided the clearest image. All subsequent sequence imaging was taken at this BW. To obtain movement traces of the articular disk and the mandibular head, the plane including the lateral condyle path of the mandibular head was selected. To obtain movement traces of the mandibular central incisor, which is the most common measuring point to measure mandibular movement, the midsagittal section was selected. Finally, to image the movement on the horizontal section of the temporomandibular joints, including the right and left mandibular heads in opening and closing the mouth, the horizontal section of the temporomandibular joint was selected (Figure 1). Four cross sections, i.e., the midsagittal section of the mandibular central incisor, the sagittal sections of the right temporomandibular joint, the sagittal sections of the left temporomandibular joint, and the horizontal section containing the heads of right and left temporomandibular joints, were sequentially excited and imaged at each slice scanning (multi-section imaging). This scanning procedure was repeated 14 times successively. The scanning time was 1.5 seconds for each scan and lasted approximately 21 seconds in total. The multi-section imaging is likely to reduce the uniformity of magnetic field in MR due to 
wide-range imaging, including the sagittal and horizontal section, resulting in a reduction in the SNR. However, reduction of the SNR was prevented by placing head, double-loop and neck coils to cover the whole mandible and allow sufficient 3D shimming.

Images were taken under the following conditions: FOV read:350 mm,FOV Phas:68.8 \%,thickness:3.0 mm,TR:5.38 Hz/Px,TE:2.69 ms, flip angle: 70 deg, ScanTime:1.6 s, Vox:1.82*1.37*3 mm, Measurements:14, Base resolution:256, Phase resolution:75 \%, Phase partial Fourier:6/8, BandWidth:592 Hz/Pix.

2. Measuring of movement of the mandibular head

[Analysis method]

In the movement of temporomandibular joints during opening and closing of the mouth, the maxilla moves slightly backward. Therefore, to examine the mandibular movement alone, measurement and reference points were established in MR images (Figure 1), and using relative coordinates, the mandibular movement at the measurement point was plotted. In tracing movement of the mandibular central incisor, the measurement point of the mandibular bone in which the mandibular central incisor root was fixed and two arbitrary reference points with a sharp contrast were also fixed in the skull to estimate the movement trace. Similarly, movement of the right and left mandibular heads was plotted by tracing the points of the right 
and left mandibular heads as the measurement points and fixing referent points around the mandibular torus and the acoustic pore. Furthermore, on the horizontal plane, the midpoint of the right and left mandibular heads was measured and movement of the point was traced and plotted by establishing the right and left external acoustic pores as the reference points. 


\section{Results}

After the true FISP sequence was optimized, three-dimensional movement of the mandibular joint during opening and closing the mouth was successively measured on four different sections. MRI using double-loop, neck and head coils was able to obtain all of the data concerning the mandible in opening and closing of the mouth; consequently, the whole mandibular movement was steadily imaged. There was no interference between coils and no artifact generated. In all volunteers, the mandibular and maxillary movement and tongue motion were observed in the midsagittal section. In the horizontal section including the right and left mandibular joints, the movement of the right and left mandibular head and the lateral pterygoid muscle were observed simultaneously. In the sagittal sections of the temporomandibular joint, the movement of the mandibular head, articular disk, the superior head of lateral pterygoid muscle and articular cavity were observed. Similarly, in the horizontal section of temporomandibular joints, including the right and left mandibular heads, in opening and closing of the mouth, the mandibular head and relaxation and contraction of the lateral pterygoid, masseter and temporal muscles were clearly observed.

Of 10 volunteers, 4 exhibited smooth linear movement of the right and left temporomandibular joints on the horizontal section of the temporomandibular joints during opening and closing of the temporomandibular joints. However, trismus due to the articular disk was found in the images of the right and left temporomandibular sagittal sections in one 
volunteer while no trismus was detected in the images of other sections in the other three volunteers. In the remaining 6 volunteers, disturbance in opening and closing of the temporomandibular joints was found in all sections, and two of them exhibited marked disorders. The details of these two volunteers are described below.

In measurement for one of the two volunteers noted above, the moving image on the sagittal section of the right temporomandibular joint showed that the mandibular head did not arc but moved discontinuously, indicating reduced flexibility in the articular disk. Similarly, the moving image on the sagittal section of the left temporomandibular joint showed reduced flexibility in the articular disk; consequently, it was confirmed that the articular disk inhibited the mandibular head from opening and did not move with the mandibular head but slipped backward. The horizontal section image indicated that the maximum change in opening and closing of the mouth was larger in the right mandibular head compared with that in the left one. The MR images on four sections during opening and closing of the mouth of this volunteer and the results of movement measurement are shown in Figure 2 to Figure 5. Figure 6 shows the moving image on the sagittal section of the right temporomandibular joint in which the joint moved along a crank during opening of the mouth. The moving image on the sagittal section of the left temporomandibular joint showed that the tracing path during opening of the mouth did not correspond to that during closure of the mouth. 
Regarding the other volunteer, the moving image on the midsagittal section of the mandible showed normal movement. However, the MR image on the sagittal section of the right temporomandibular joint showed trismus disturbed by the articular disk and the articular disk slipped backward during opening of the mouth. However, the MR image on the sagittal section of the left temporomandibular joint showed that the articular disk was dislocated forward and that the articular disk returned to the original position during opening of the mouth and was dislocated forward during closure of the mouth. The horizontal section image of the mandible showed no difference in the maximum change in opening and closing of the mouth between the right and left mandibular heads and it was confirmed that the right and left mandibular heads moved in parallel. The MR images on four sections during opening and closing of the mouth in this volunteer and the results of movement measurement are shown in Figure 7.

The MR image on the sagittal section of the right temporomandibular joint showed trismus disturbed by the articular disk and the articular disk slipped backward during opening of the mouth, which corresponded to the horizontal movement during the latter half of mouth-opening, as shown in Figure 7. The MR image on the sagittal section of the left temporomandibular joint showed that the articular disk was dislocated forward and that the articular disk returned to the original position during opening of the mouth and was dislocated forward during closure of the mouth, which was shown in Figure 7. The horizontal section 
image of the mandible showed no difference in the maximum change during opening and closing of the mouth between the right and left mandibular heads and it was confirmed that the right and left mandibular heads moved in parallel. 


\section{Discussion}

\section{Multi-section MR imaging}

A study using fast MRI reported a semi-moving image of the temporomandibular joint in one section. However, the study used a series of images of the joint in several still conditions, not a natural movement of opening and closing of the mouth[7]. To accurately understand movement of the temporomandibular joint, a series of still images can be useful, but continuous images in the moving condition is optimal.

To continuously image the moving mandible and actually obtain measurable images, it is necessary to take MR images without reducing the spatial and density resolution. We previously imaged the temporomandibular joints during opening and closing of the mouth without stopping and obtained continuous images with TR, BW and FOV optimized by ultrafast imaging Turbo-FLASH sequence, and measured the mandibular movement[12, 17]. In that study, however, the right and left temporomandibular joints were imaged separately. The temporomandibular joint is the only bilaterally coupled joint in the human body and is a special joint that makes gliding and rotating movements simultaneously. The movement of the one temporomandibular joint has an effect on that of the other one and both joints interact each other. Therefore, for accurate measurement of the movement of the temporomandibular joint, it was considered necessary to measure the right and left temporomandibular joints during opening and closing of the same mouth. 
The Turbo FLASH is a single-imaged sequence in which a preparation pulse is added to a gradient-echo FLASH (Fast Low Angle Shot) with shortened TE and TR in order to enhance the contrast. This results in an ultrafast imaging sequence although its spatial resolution is not high. One problem with this approach is that shortening of the TR to decrease the imaging time induces a reduction in the contrast between tissues. In this study, to maintain sufficient SNR, the True FISP sequence was optimized and multiple types of coils were used.

The True FISP sequence is a sequence in which a short TR $(<\mathrm{T} 1, \mathrm{~T} 2)$ makes a steady -state with transverse and longitudinal magnetization and continuously generates MR signals of the same magnitude (steady -state movement with free precession). Consequently, steady -state MR signals provide images with good SNR even in a short imaging time. In this study, the optimized True FISP sequence provided increased imaging sections with the optimal TR. Consequently, multi-section images of the right and left temporomandibular joints in the same movement were obtained during opening and closing of the mouth. In addition, continuous imaging on the sagittal and horizontal sections was conducted by 3D shimming and uniform magnetic field using plural type coils. A decreased TR reduces the phase error associated with less binding artifacts.

However, in preliminary imaging, the image of the temporomandibular joints was clearest with $\mathrm{TR}=5.38 \mathrm{~ms}$ and $\mathrm{BW}=305 \mathrm{~Hz} / \mathrm{ZPx}$, but not $\mathrm{TR}=3.6 \mathrm{~ms}$, the lowest value, or $\mathrm{BW}=1502$ $\mathrm{Ha} / \mathrm{Px}$. One possible reason is that in multi-section imaging four different sections were 
successively imaged during one scan and a disturbance in the magnetic field by imaging of a certain section had an effect on imaging of the next section due to the shortened imaging time, so that images of the temporomandibular joints were clearest with the condition of $\mathrm{TR}=5.38$ ms and $\mathrm{BW}=305 \mathrm{~Hz} / \mathrm{Px}$.

\section{Mandibular movement}

To measure the temporomandibular joint comprising hard and soft tissues, MRI that simultaneously measures the internal structure of hard and soft tissues can be extremely useful; distinct from a measurement device that measures hard tissues, such as the device for mandibular movement measuring at the point of the mandibular incisor. The measurement results of this study in a volunteer with marked trismus confirmed that the left mandibular head stopped but the right one did not; consequently, the mandible was rotated in the left direction as the movement continued on the horizontal section. Ultrafast MRI of the same mandibular movement provides not only sagittal but also horizontal-section images of the right and left temporomandibular joints; i.e., the temporomandibular joint dysfunction can be observed in three directions.

MRI provides images that show the type and extent of soft tissues of the articular disk; consequently, it demonstrates that the movement of the articular disk has an effect on the 
mandibular movement. However, it cannot show the path that the mandibular head actually makes; therefore, it is important to analyze the movement using MR images. MR images that can show the soft tissue and allow analysis of the mandibular movement are complementary and their combination can provide for comprehensive diagnosis.

Simultaneous multi-section measurement of movement of the temporomandibular joints provides simple 3D analysis of the joints. This method can be applied not only to the temporomandibular joints but also various joints including the elbow and knee. In addition, as was performed in this study, quantitative measurement of a trace by MR images with established numerical parameters for the moving region has the potential to be helpful for doctors in clinical observation and diagnosis for moving tissues, including temporomandibular dysfunction. Therefore, this multi-section imaging method is a very effective method that easily displays 3-dimensional movement. 


\section{Reference}

[1]. Jemt TandHedegard B. Reproducibility of chewing rhythm and of mandibular displacements during chewing. J Oral Rehabil 1982; 9:531-537.

[2]. Mongini FandTempia-Valenta G. A graphic and statistical analysis of the chewing movements in function and dysfunction. J Craniomandibular Pract 1984; 2:125-134.

[3]. Proschel PandHofmann M. Frontal chewing patterns of the incisor point and their dependence on resistance of food and type of occlusion. J Prosthet Dent 1988; 59:617-624.

[4]. Fushima K, Sato S, Suzuki YandKashima I. Horizontal condylar path in patients with disk displacement with reduction. Cranio 1994; 12:78-86; discussion 87.

[5]. Katzberg RW, Schenck J, Roberts D, et al. Magnetic resonance imaging of the temporomandibular joint meniscus. Oral Surg Oral Med Oral Pathol 1985; 59:332-335.

[6]. Tasaki MM, Westesson PLandRaubertas RF. Observer variation in interpretation of magnetic resonance images of the temporomandibular joint. Oral Surg Oral Med Oral Pathol 1993; 76:231-234.

[7]. Drace JEandEnzmann DR. Defining the normal temporomandibular joint: closed-, partially open-, and open-mouth MR imaging of asymptomatic subjects. Radiology 1990; 177:67-71.

[8]. Shellock FG, Mink JH, Deutsch AandPressman BD. Kinematic magnetic resonance imaging of the joints: techniques and clinical applications. Magn Reson Q 1991; 7:104-135.

[9]. Azuma T, Nambu T, Doi K, et al. Motion Analysis of Mandible with Turbo-FLASH MR Imaging System. Journal of Japanese Society for Clinical Biomechanics 2000; 21:489-495.

[10]. Maniere-Ezvan A, Havet T, Franconi JM, Quemar JCandde Certaines JD. Cinematic study of temporomandibular joint motion using ultra-fast magnetic resonance imaging. Cranio 1999; 17:262-267.

[11]. Chen YJ, Gallo LM, Meier DandPalla S. Dynamic magnetic resonance imaging technique for the study of the temporomandibular joint. J Orofac Pain 2000; 14:65-73.

[12]. Quick HH, Ladd ME, Hoevel M, et al. Real-time MRI of joint movement with trueFISP. J Magn Reson Imaging 2002; 15:710-715.

[13]. Shimazaki Y, Saito K, Matsukawa S, et al. Image quality using dynamic MR imaging of the temporomandibular joint with true-FISP sequence. Magn Reson Med Sci 2007; 6:15-20.

[14]. Zhang XN, Nishiyama H, Murakami SandFuchihata H. A study of condylar movement by bilateral simultaneous videofluorography. Dentomaxillofac Radiol 1998; 27:279-286.

[15]. Sanchez-Woodworth RE, Tallents RH, Katzberg RWandGuay JA. Bilateral internal derangements of temporomandibular joint: evaluation by magnetic resonance imaging. Oral Surg Oral Med Oral Pathol 1988; 65:281-285.

[16]. Gallo LM. Modeling of temporomandibular joint function using MRI and jaw-tracking technologies--mechanics. Cells Tissues Organs 2005; 180:54-68.

[17]. Basser PJandPajevic S. Statistical artifacts in diffusion tensor MRI (DT-MRI) caused by background noise. Magn Reson Med 2000; 44:41-50. 


\section{Figure and Legends}

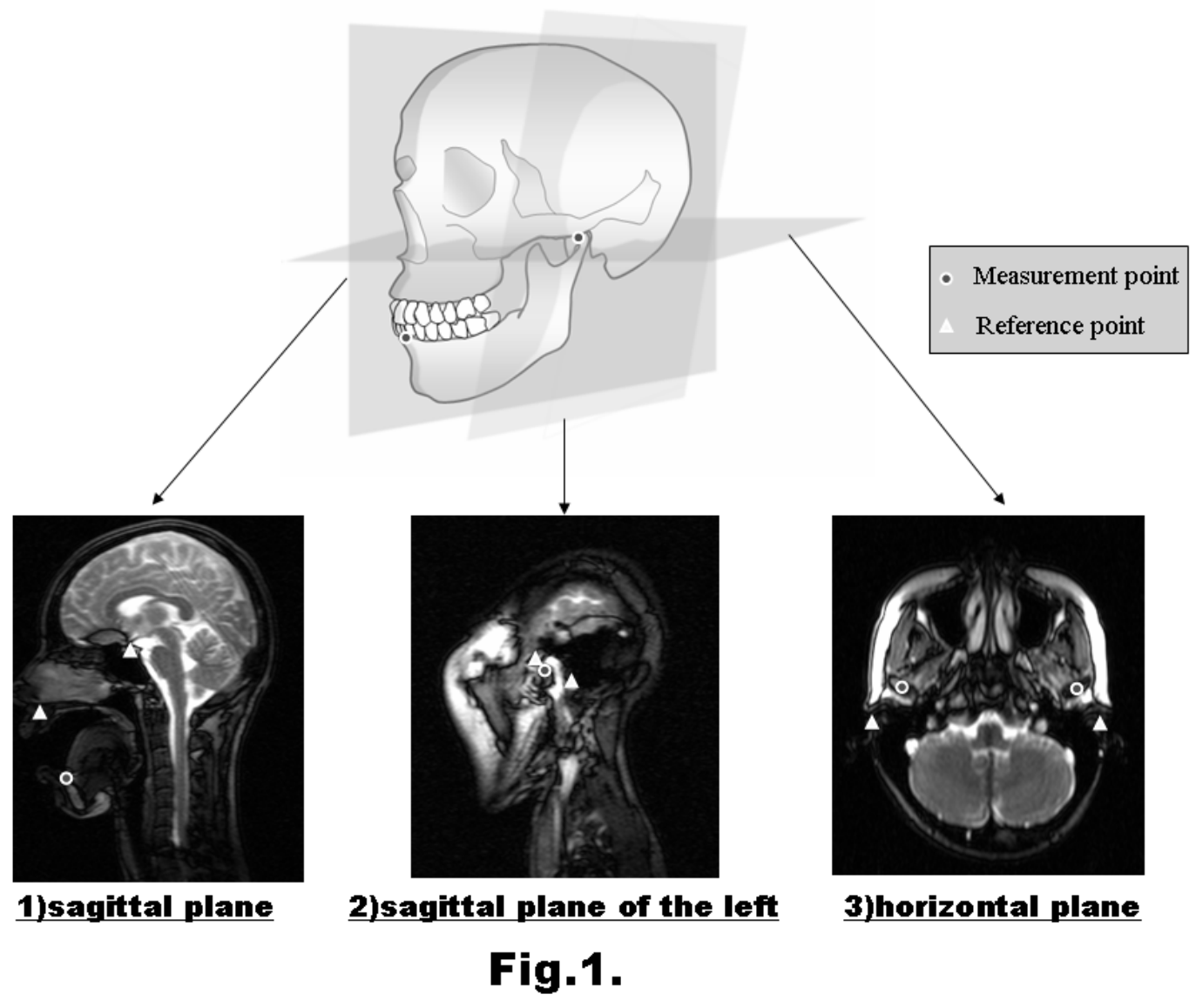

Figure 1. MR images on four different types of sections: 1) the midsagittal section of the mandible - a movement trace of the mandibular central incisor, which is the most common measuring point to measure mandibular movement. 2) The midsagittal section of the temporomandibular joint - a movement trace of the lateral condyle path of the mandibular head to obtain movement traces of the mandibular head. 3) The horizontal section of the temporomandibular joint - a horizontal movement trace of the right and left mandibular heads. Measurement and reference points were indicated by $\circ$ and $\Delta$, respectively, in each image. 

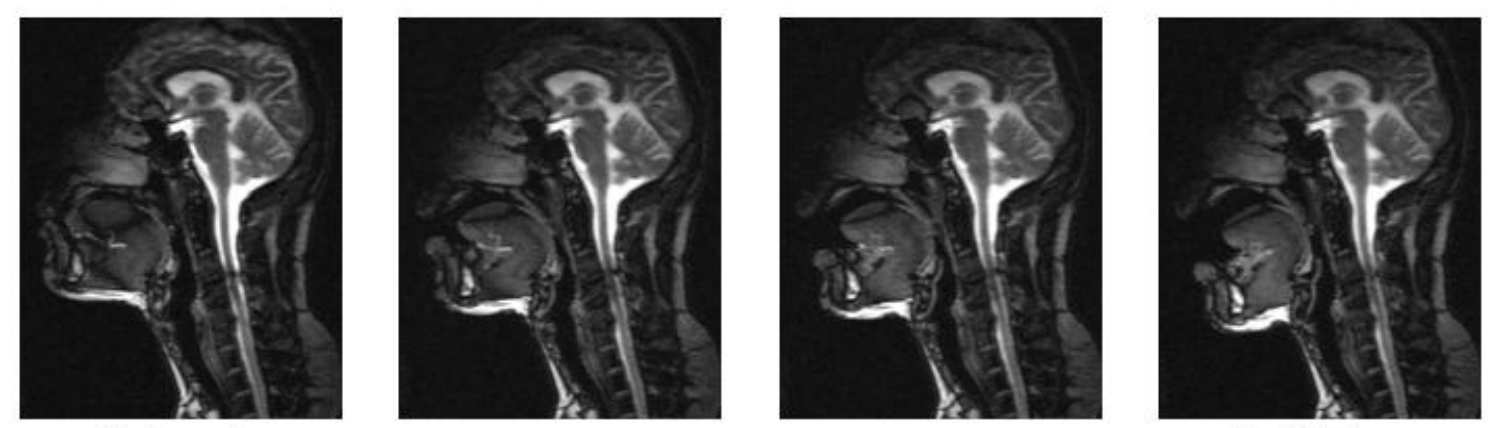

Start
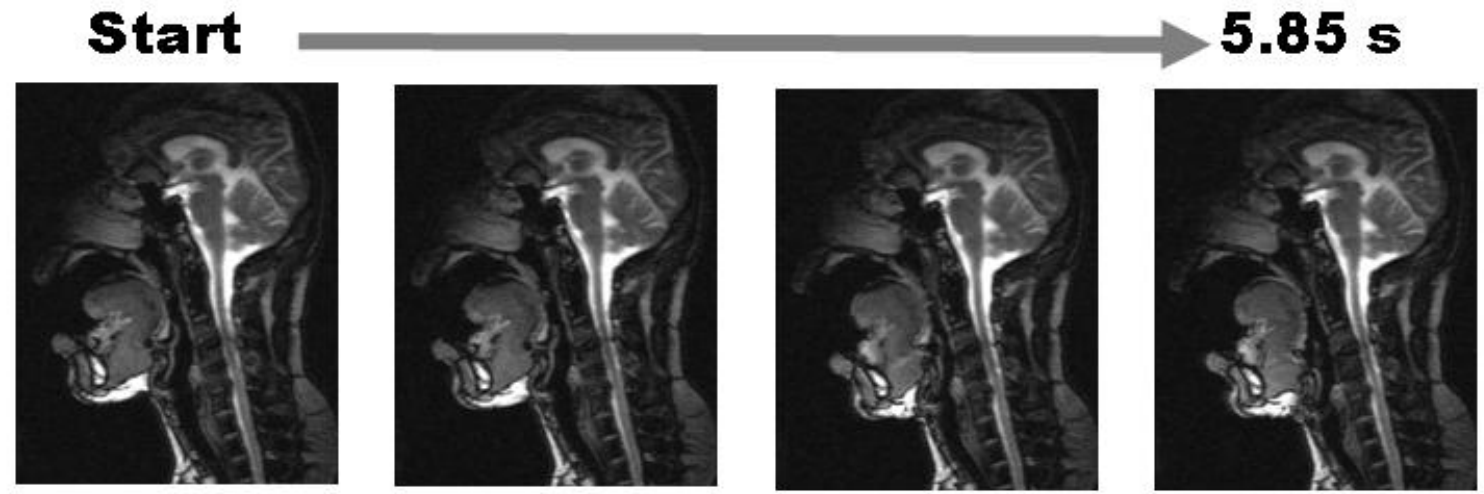

7.2 s
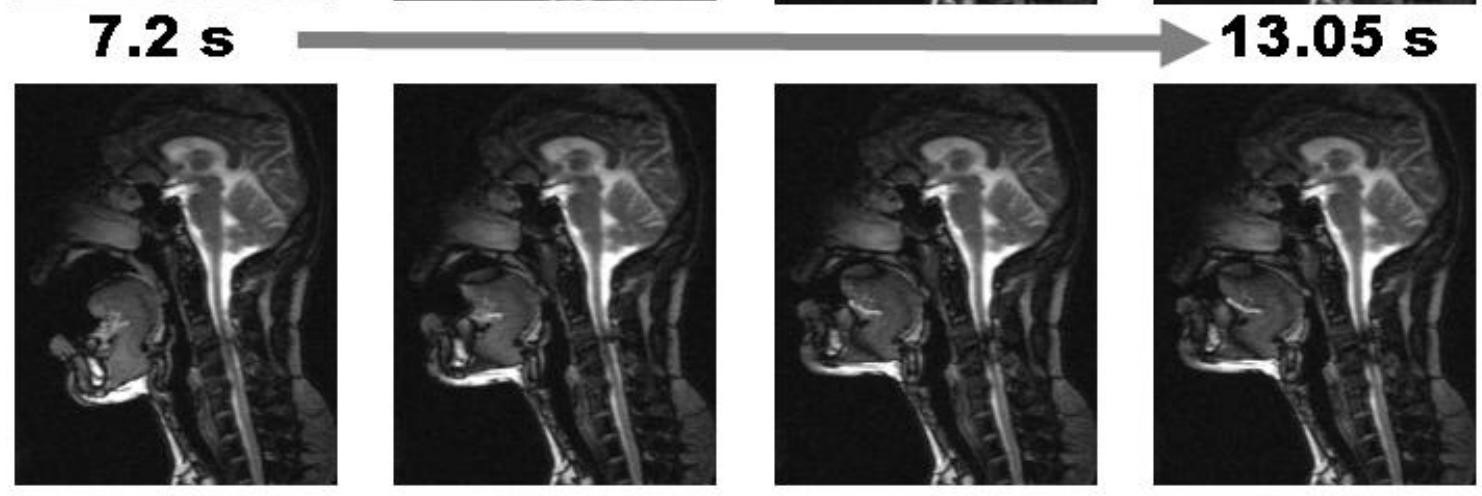

$14.4 \mathrm{~s}$

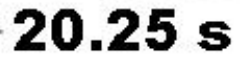

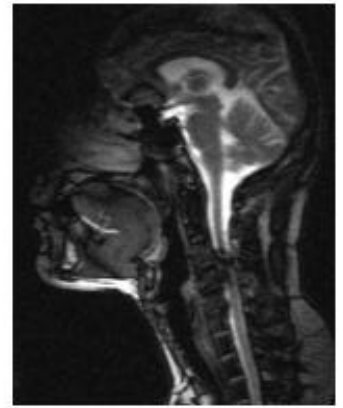

$21.6 \mathrm{~s}$

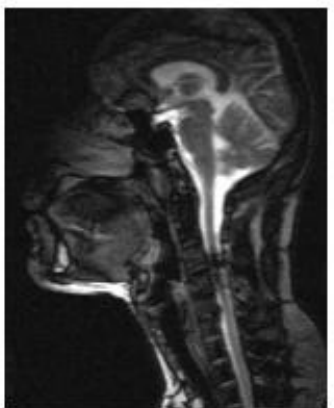

23.85 s
Fig.2.

Figure 2. Successive MR images of the mandible on the sagittal section during opening and closing of the mouth of a volunteer with marked trismus. 

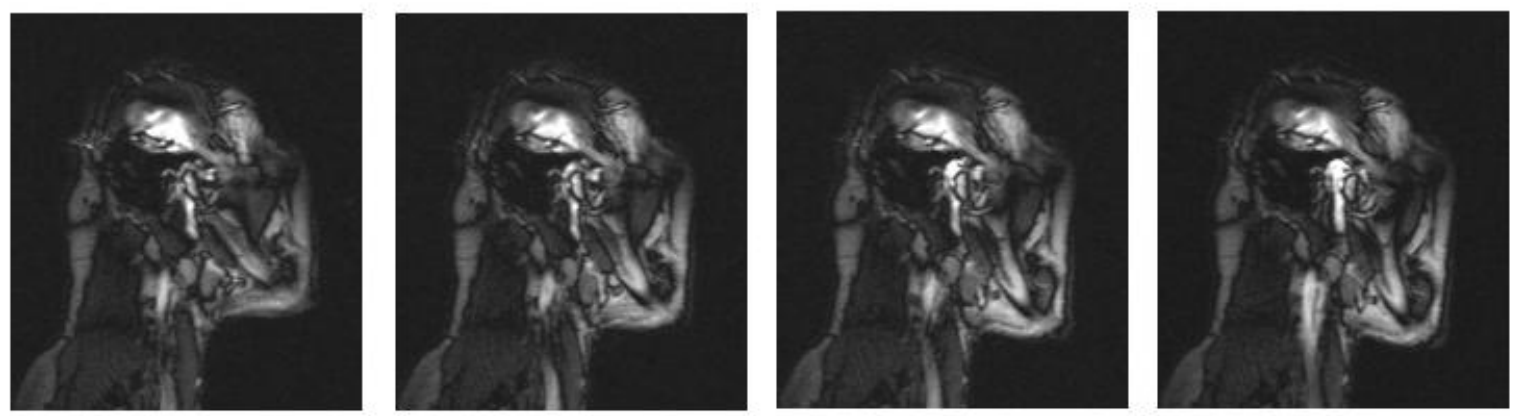

Start

$5.85 \mathrm{~s}$
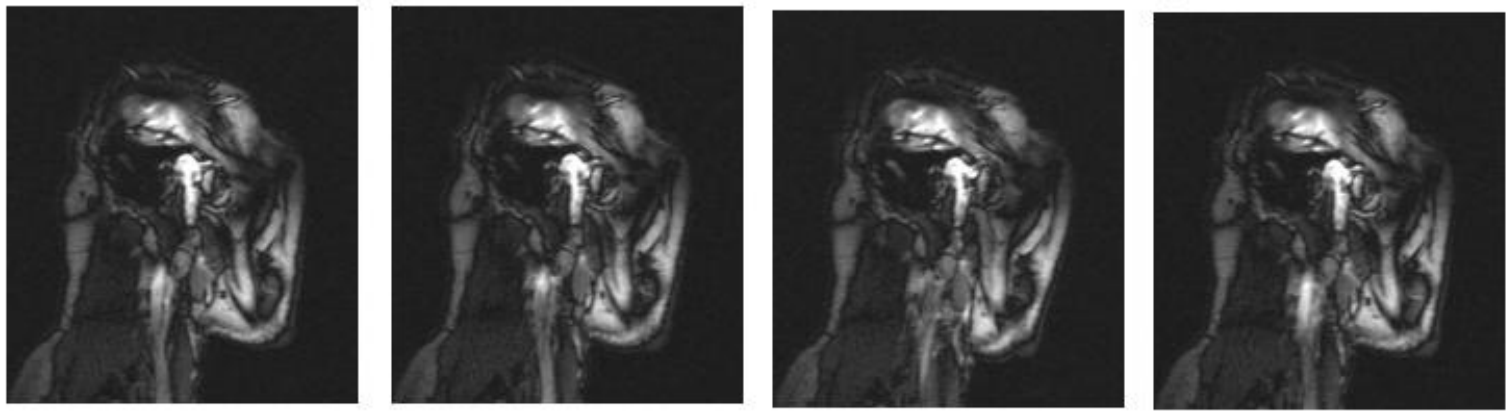

$7.2 \mathrm{~s}$

$13.05 \mathrm{~s}$
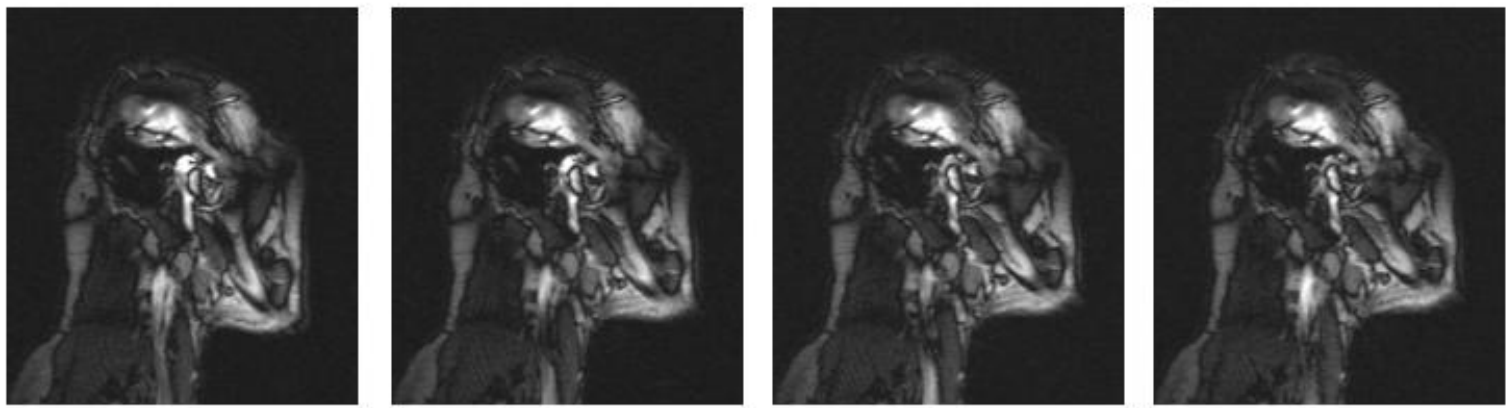

$14.4 \mathrm{~s}$

\section{$20.25 \mathrm{~s}$}

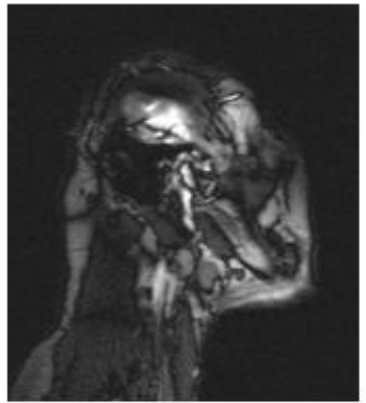

$21.6 \mathrm{~s}$
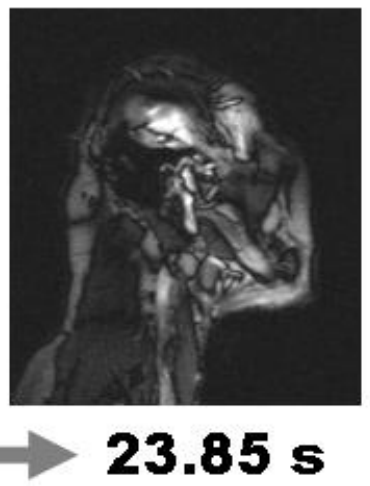

Fig.3.

Figure 3. Successive MR images of the right temporomandibular joint in the sagittal section during opening and closing of the mouth of a volunteer with marked trismus. 

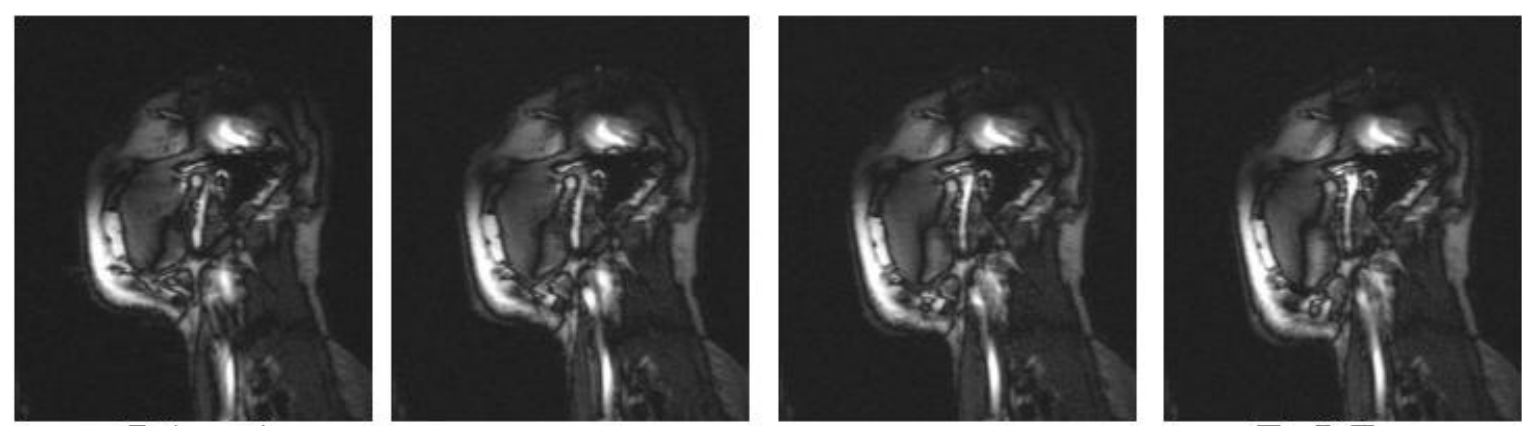

Start
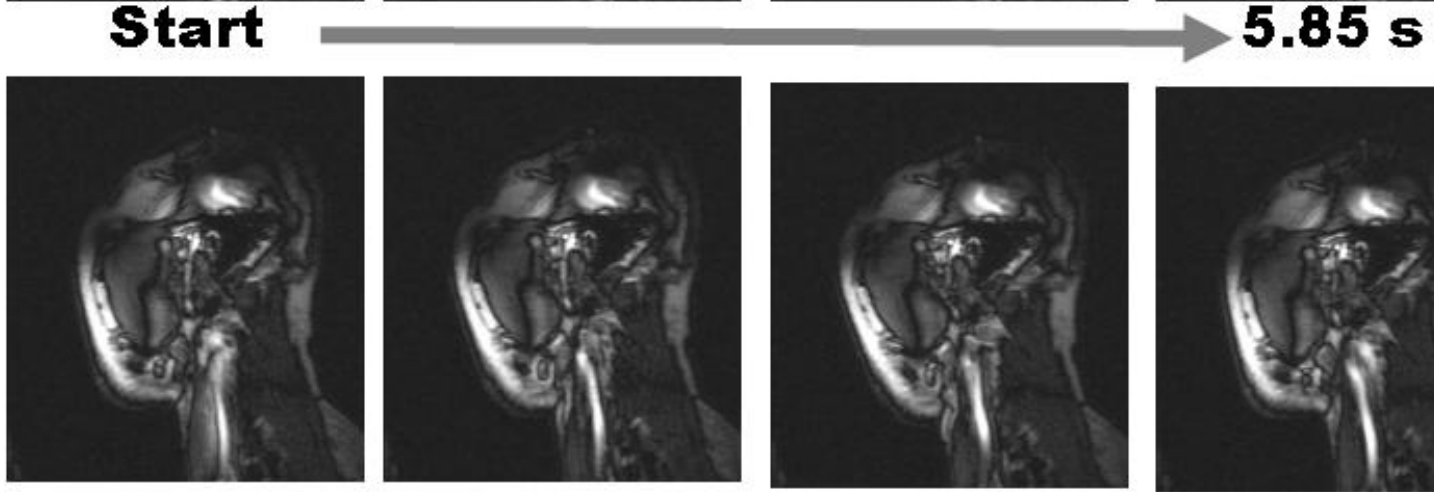

$7.2 \mathrm{~s}$
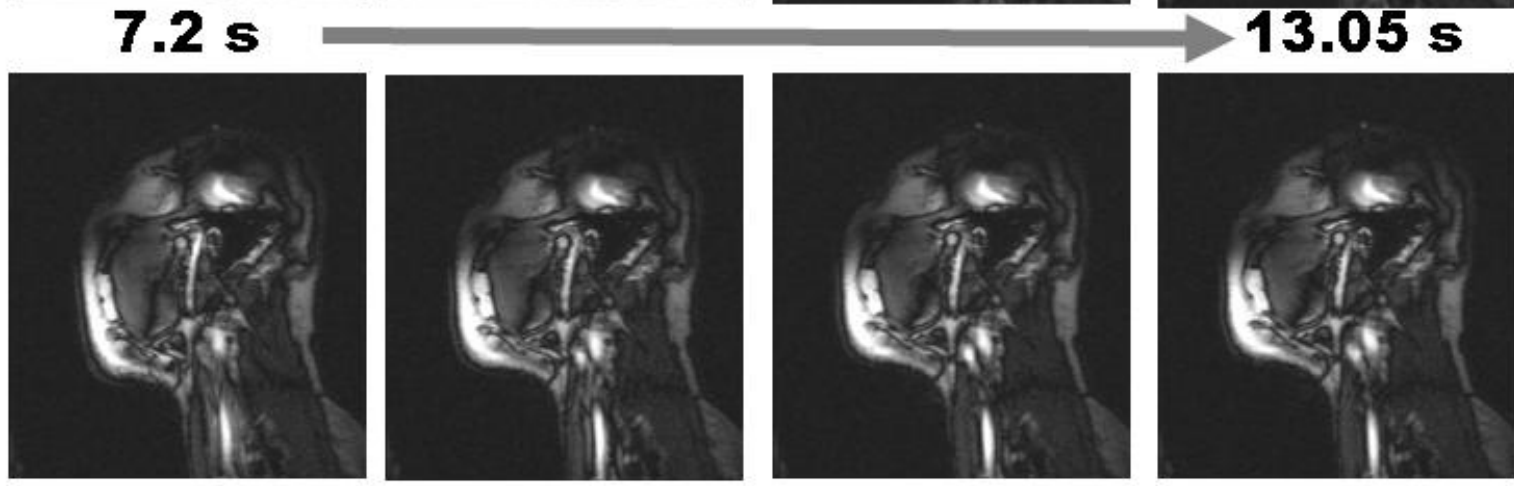

$14.4 \mathrm{~s}$
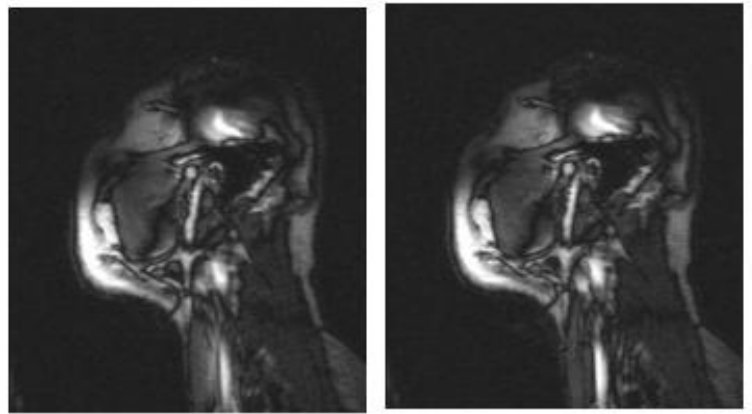

$21.6 \mathrm{~s}$

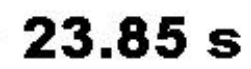

Fig.4.

Figure 4. Successive MR images of the left temporomandibular joint on the sagittal section during opening and closing of the mouth of the volunteer with marked trismus. It is confirmed that the articular disk reduced flexibility and inhibited the mandibular head during 
opening; consequently, it did not move with the mandibular head but slipped backward.
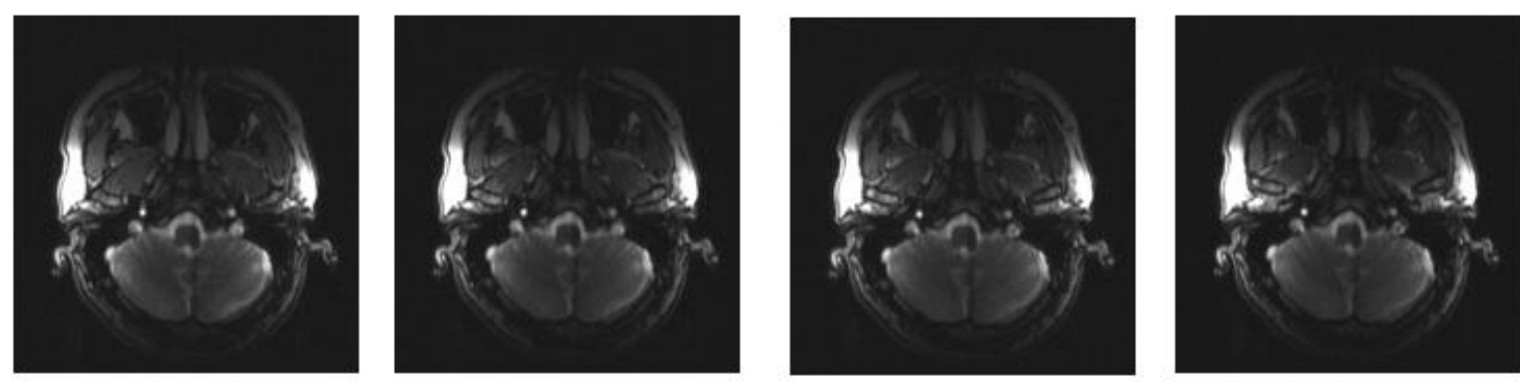

Start
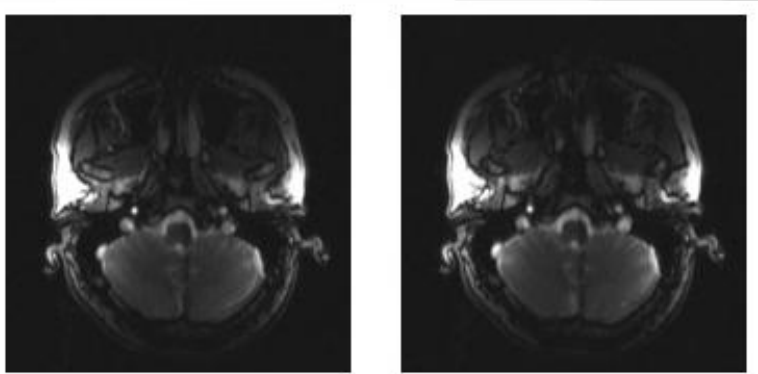

$5.85 \mathrm{~s}$

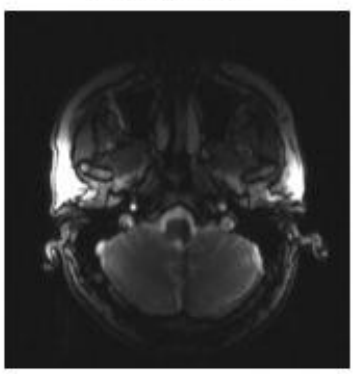

$7.2 \mathrm{~s}$
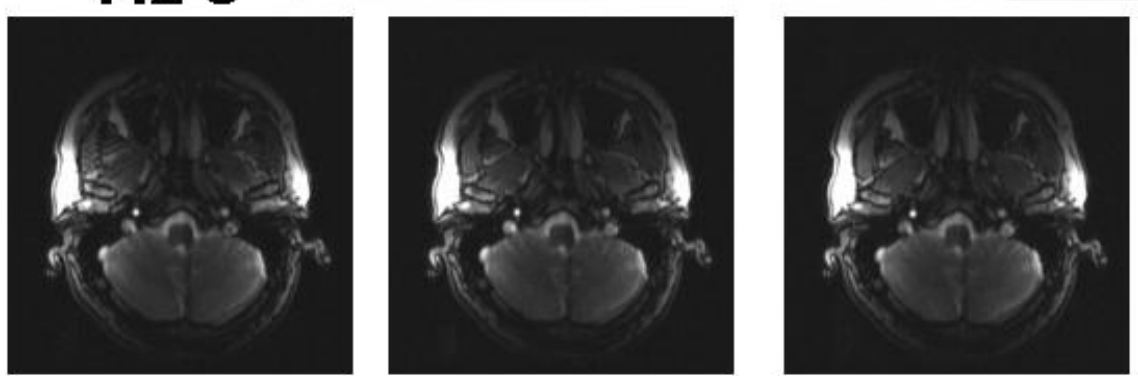

$13.05 \mathrm{~s}$

$14.4 \mathrm{~s}$
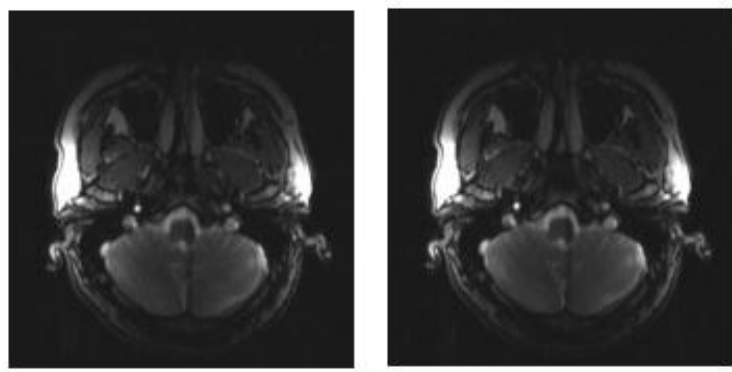

$21.6 \mathrm{~s}$

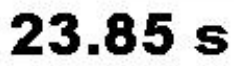

Fig.5.

Figure 5. Successive MR images of the right and left temporomandibular joints on the horizontal section during opening and closing of the mouth of the volunteer with marked trismus. It is indicated that the maximum change during opening and closing of the mouth 
was larger in the right mandibular head than that in the left one.
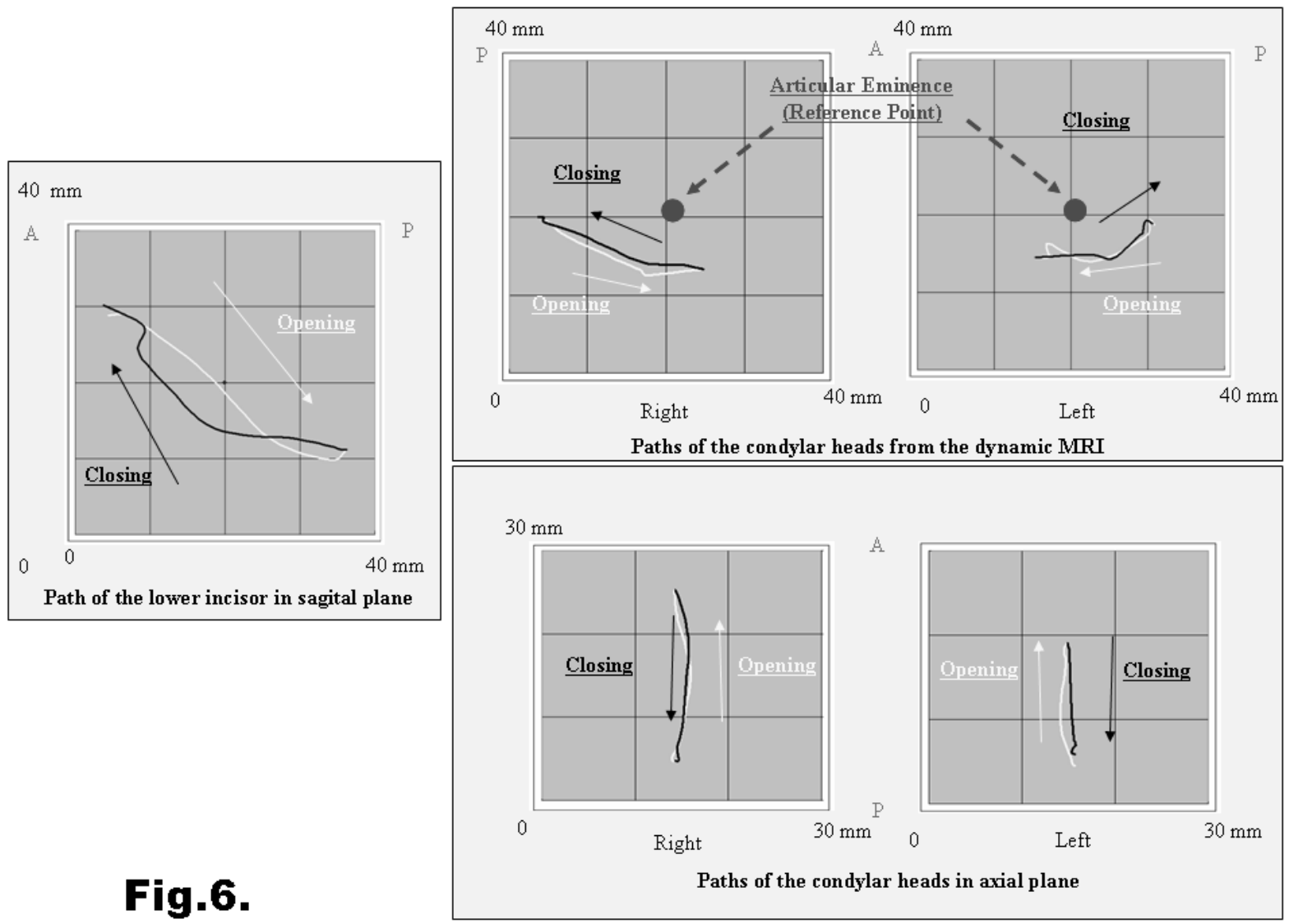

Fig.6.

Figure 6. The movement traces of the mandibular central incisor and the peak of the mandibular head are shown based on successive MR images of the mandible on the sagittal section and the right and left temporomandibular joints on the sagittal and horizontal sections in opening and closing of the mouth of the volunteer with marked trismus. The MR images of the right temporomandibular joint on the sagittal section (Figures 2 to 5) show that the mandibular head did not arc but moved discontinuously, indicating reduced flexibility in the articular disk and that the joint moved in crank-shaped manner in opening the mouth. The 
sagittal section image of the left temporomandibular joint shows reduced flexibility in the articular disk, consequently the articular disk inhibited the mandibular head from opening and did not move with the mandibular head but slipped backward, indicating that a line in opening the mouth did not correspond to that in closing the mouth. The graph of the horizontal movement indicates that the maximum change in opening and closing of the mouth was larger in the right mandibular head than that in the left one.
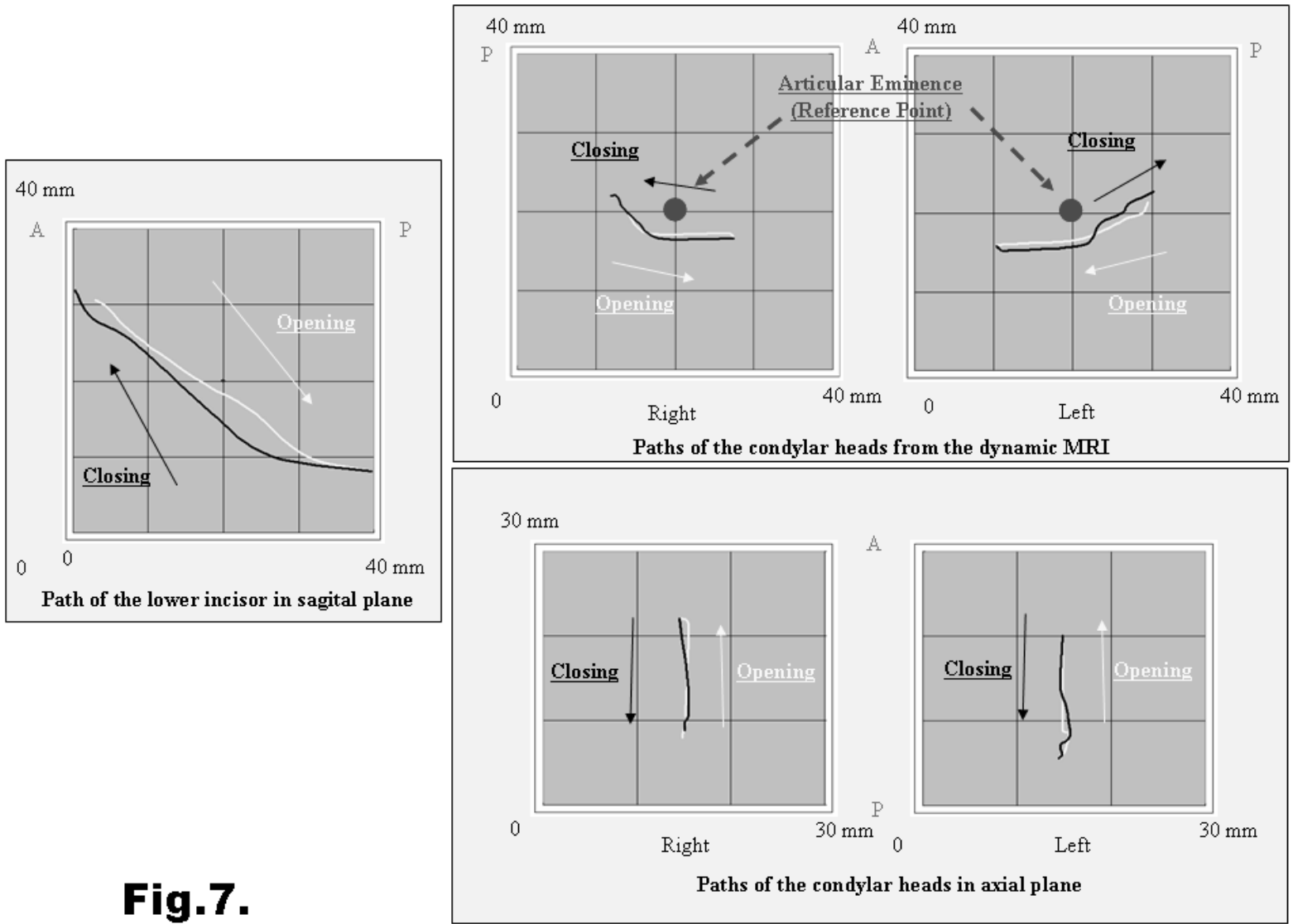

Fig.7.

Figure 7. The movement traces of the mandibular central incisor and the peak of the mandibular head are shown based on the successive MR images of the mandible on the 
sagittal section and the right and left temporomandibular joints on the sagittal and horizontal sections in opening and closing of the mouth of the volunteer with normal movement on the midsagittal section. The MR image on the sagittal section of the right temporomandibular joint shows trismus disturbed by the articular disk and that the articular disk slipped backward during opening of the mouth. The above movement corresponds to the horizontal part of movement during the latter half of mouth-opening in the graph. The MR image on the sagittal section of the left temporomandibular joint shows that the articular disk was dislocated forward and that the articular disk returned to the original position during opening of the mouth and was dislocated forward during closing of the mouth. The graph shows a movement trace indicating that the articular disk returned to the original position. The horizontal section image of the mandible showed no difference in the maximum change in opening and closing of the mouth between the right and left mandibular heads and it was confirmed that the right and left mandibular heads moved in parallel. 